\title{
The Positive Effects of Hydrophobic Fluoropolymers on the Electrical Properties of $\mathrm{MoS}_{2}$ Transistors
}

\author{
Somayyeh Rahimi ${ }^{1,2,+}$, Rudresh Ghosh ${ }^{2,+, *}$, Seohee Kim ${ }^{1,2}$, Ananth Dodabalapur ${ }^{1,2}$, \\ Sanjay Banerjee ${ }^{1,2}$ and Deji Akinwande ${ }^{1,2}$ \\ 1 Department of Electrical and Computer Engineering, University of Texas at Austin, Austin, TX 78758, USA; \\ nrahimi@gmail.com (S.R.); seohee@utexas.edu (S.K.); ananth.dodabalapur@engr.utexas.edu (A.D.); \\ banerjee@ece.utexas.edu (S.B.); deji@ece.utexas.edu (D.A.) \\ 2 Microelectronics Research Center, University of Texas at Austin, Austin, TX 78758, USA \\ * Correspondence: rudresh@utexas.edu; Tel.: +1-614-620-1855 \\ + These authors contributed equally to this work.
}

Academic Editor: Andres Castellanos-Gomez

Received: 19 July 2016; Accepted: 12 August 2016; Published: 23 August 2016

\begin{abstract}
We report the improvement of the electrical performance of field effect transistors (FETs) fabricated on monolayer chemical vapor deposited (CVD) $\mathrm{MoS}_{2}$, by applying an interacting fluoropolymer capping layer (Teflon-AF). The electrical characterizations of more than 60 FETs, after applying Teflon-AF cap, show significant improvement of the device properties and reduced device to device variation. The improvement includes: $50 \%$ reduction of the average gate hysteresis, $30 \%$ reduction of the subthreshold swing and about an order of magnitude increase of the current on-off ratio. These favorable changes in device performance are attributed to the reduced exposure of $\mathrm{MoS}_{2}$ channels to the adsorbates in the ambient which can be explained by the polar nature of Teflon-AF cap. A positive shift in the threshold voltage of all the measured FETs is observed, which translates to the more desirable enhancement mode transistor characteristics.
\end{abstract}

Keywords: transition metal dichalcogenides; 2-Dimensional materials; molybdenum disulfide; chemical vapor deposition; field effect transistors; fluoropolymer; passivation

\section{Introduction}

Two dimensional atomic solids such as graphene and transition metal dichalcogenides (TMDs) have attracted a great amount of interest due to their thickness-dependent electrical and optical properties. Of TMDs, Molybdenum disulfide, $\left(\mathrm{MoS}_{2}\right)$, with its indirect to direct band gap transition as a function of layer thickness has been of particular interest for digital and optoelectronic applications [1-4]. $\mathrm{MoS}_{2}$ has already been used to fabricate functional electronic circuit elements [5,6], as well as opto-electronic devices [7], and coupled electro-mechanic systems [8,9]. Recent research has also shown its applicability for high frequency applications $[10,11]$. Also, the recent progress towards the wafer-scale growth and batch fabrication of field-effect transistors (FETs) on chemical vapor deposited (CVD) monolayer 2D solids such as graphene [12] and recently $\mathrm{MoS}_{2}$ and $\mathrm{WS}_{2}$ [13], has shown great promise for realizing commercial applications of these materials.

However, the performance of FETs fabricated on these wafer-scale films are largely impacted by device to device variability, including gate hysteresis and threshold voltage variations, e.g. threshold voltage of $\sim 100$ tested $\mathrm{MoS}_{2}$ FETs, fabricated on 100-mm silicon substrates, ranges between $10 \mathrm{~V}$ and $45 \mathrm{~V}$ with the reported mobility in $1-40 \mathrm{~cm}^{2} / \mathrm{Vs}$ range and high variability in the current on-off ratio and gate hysteresis. This significant performance variability are often caused by the charges trapped between the 2D device channel and the gate dielectric as well as oxygen and moisture chemisorption at the basal plane and edges of $\mathrm{MoS}_{2}$ [14-17]. Therefore, one way to reduce performance variability 
and gate hysteresis is taking the measurements under vacuum or long-term annealing of $\mathrm{MoS}_{2}$ FETs at elevated temperatures prior to testing the device [18]. Both of these techniques leads to removing the adsorbed oxygen/moisture content from the $\mathrm{MoS}_{2}$ channel surface. A second method to reduce the channel interaction with the ambient, is to apply hydrophobic capping layers to the top surface of the device. Previous reports have shown that the performance variations of graphene and carbon nanotube (CNT) FETs can be reduced by applying a monolayer self-assembled hexamethyldisilazane (HMDS) layer between the back gate dielectric and the device channel or on the top surface of the device channels [18-20]. Other reports showed that passivating the surface of graphene and phosphorene FETs by a thicker hydrophobic film, such as fluoropolymers, results in more lasting positive changes and improved consistency in device to device performance [21-23].

Here, we report the impact of encapsulation of $\mathrm{MoS}_{2}$ FETs by a hydrophobic fluoropolymer, Teflon-AF. Our results show significant improvement in key device performance metrics such as current on-off ratio, subthreshold swing and gate hysteresis. Key performance metrics, such as threshold voltage, subthreshold swing, gate hysteresis and on-off ratio also show improved consistency after applying the fluoropolymer cap.

\section{Materials and Methods}

All of the devices of this work were fabricated on P+ silicon bottom gate with $300 \mathrm{~nm}$-thick thermally grown $\mathrm{SiO}_{2}$ that was used as the gate dielectric. Monolayer $\mathrm{MoS}_{2}$ was grown on the substrate using a CVD process explained elsewhere [10]. An optical images of CVD $\mathrm{MoS}_{2}$ crystal grown on $300 \mathrm{~nm} \mathrm{SiO}$ is shown in Figure 1a. The monolayer structure of the grown CVD films were confirmed by Raman and photoluminescence (PL) spectroscopy shown in Figure 2. The $E_{2 g}^{1}$ and $A_{1 g}$ peaks are at $389.3 \mathrm{~cm}^{-1}$ and $409.1 \mathrm{~cm}^{-1}$, respectively. This corresponds to a peak separation of $19.8 \mathrm{~cm}^{-1}$, which is characteristic of single-layer $\mathrm{MoS}_{2}$ [24]. The PL spectrum of the CVD MoS 2 also shows a strong peak at around $685 \mathrm{~nm}$ which corresponds to monolayer $\mathrm{MoS}_{2}$ [25].
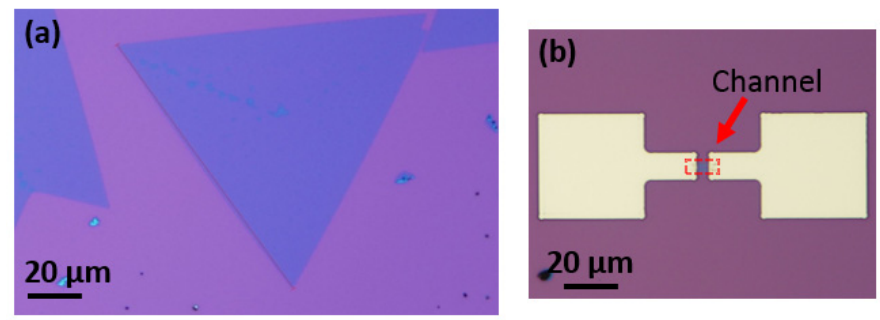

Figure 1. (a) Optical microscope (Olympus, Tokyo, Japan) image of chemical vapor deposited (CVD) $\mathrm{MoS}_{2}$ crystals; (b) An image of the $\mathrm{MoS}_{2}$ field effect transistors (FET) with channel dimensions of $3 \mu \mathrm{m} \times 9 \mu \mathrm{m}$.

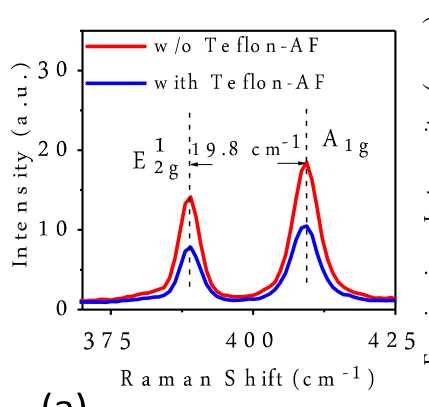

(a)

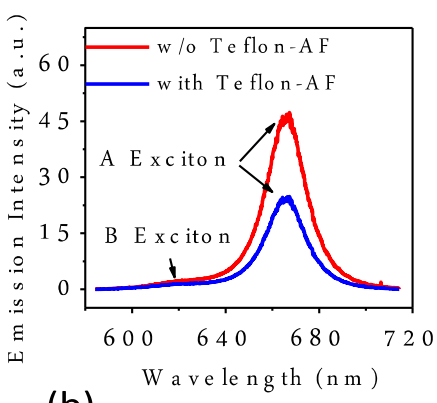

(b)

Figure 2. (a) Raman spectrum of $\mathrm{MoS}_{2}$ before (red) and after (blue) applying Teflon-AF encapsulation layer; (b) photoluminescence (PL) spectrum of monolayer $\mathrm{MoS}_{2}$ before (red) and after (blue) applying the cap. 
A conventional photolithography process was used to define the channels and contacts. The channels were isolated using $\mathrm{Cl}_{2}$ plasma followed by patterning and deposition of source/drain contacts $(20 \mathrm{~nm} \mathrm{Ag} / 20 \mathrm{~nm} \mathrm{Pd})$. The fabricated two-probe devices have a constant width of $3 \mu \mathrm{m}$ and a variable length of $3 \mu \mathrm{m}, 6 \mu \mathrm{m}$ and $9 \mu \mathrm{m}$. An optical image of the $\mathrm{MoS}_{2}$ FET with channel dimensions of $3 \mu \mathrm{m} \times 9 \mu \mathrm{m}$, is shown in Figure $1 \mathrm{~b}$.

The initial electrical characteristics of about 60 randomly-chosen devices distributed on a $5 \mathrm{~mm} \times 5 \mathrm{~mm}$ surface area were tested under room ambient conditions. The field-effect modulation of CVD MoS 2 FETs were studied by sweeping the back-gate bias in $[-100,100] \mathrm{V}$ range. Afterwards, the sample was held in $\mathrm{N}_{2}$ glovebox for half an hour, before being coated with Teflon-AF (Dupont Co., Wilmington, NC, USA). Vacuum and $\mathrm{N}_{2}$ environment were previously reported to be equally efficient in removing the adsorbates from unpassivated back-gated $\mathrm{MoS}_{2}$ devices [26]. In the next step, $140 \mathrm{~nm}$ Teflon-AF encapsulation cap was spin-coated on the sample with as-supplied Teflon-AF solution at $2000 \mathrm{rpm}$ for $1 \mathrm{~min}$. The sample was then placed on the hotplate and cured at $250{ }^{\circ} \mathrm{C}$ for $30 \mathrm{mins}$ in $\mathrm{N}_{2}$ ambient. After the cure step, the hotplate temperature was reduced to room temperature with a ramp rate of $20^{\circ} \mathrm{C} / \mathrm{min}$. Teflon-AF is a soluble fluoropolymer in selected solvents, based on copolymers of 2,2-bistrifluoromethyl-4,5-difluoro-1,3-dioxole (PDD) and tetrafluoroethylene (TFE) [27]. Teflon-AF is known to have a high-polarity amorphous structure, which is originated from a number of C-F bonds in it, with a dipole moment of 1.13D [28]. Raman and photoluminescence characterization were used to check any structural and material changes in $\mathrm{MoS}_{2}$ monolayer after applying the Teflon-AF cap. Figure 2a,b compares the Raman and PL scattering of $\mathrm{MoS}_{2}$ before and after applying the cap. The attenuated intensity of signal in both cases is likely due to the thickness of the cap.

\section{Results}

Figure 3a,b compares the electrical transfer characteristics of 15 representative devices before and after treating the surface with Teflon-AF. No drain saturation current is observed in both cases due to the thick back gate dielectric. A large variability of the device performance is observed in Figure 3a before applying the Teflon cap. This variability, which may be caused by the charges trapped between the channel and gate dielectric or the moisture and oxygen molecules adsorbed on the surface of channel, is reduced in Figure $3 b$ after applying the cap. These changes can be explained by first, the hydrophobic nature of Teflon-AF that significantly reduces the charges adsorbed on the channel surface. Second, neutralization of charge traps, within the channel and at the interface of channel and gate dielectric, by the local electric field induced in Teflon-AF. This local electric field could screen the scattering impact of trapped charges between the channel and gate dielectric. The impact of the reduction of adsorbates on the surface of the channel shows up in the form of reduced gate hysteresis, observed in all tested devices and presented in Figure 4a. For the specific device presented here, the hysteresis is reduced from $\sim 25 \mathrm{~V}$ to $\sim 10 \mathrm{~V}$. The reduction of the gate hysteresis has been reported previously for devices tested under vacuum and those tested after long-term vacuum annealing steps. Note that in none of these cases the hysteresis is reduced to zero. This is believed to be due to the remaining oxygen to be strongly bonded to the defects and edges of the $\mathrm{MoS}_{2}$ channel [18]. Improvement in the off-current states upon coating with Teflon-AF is possibly due to the polar molecules of the fluoropolymer which will partially neutralize charged dopants [22,29].

Figure $4 \mathrm{~b}$ compares the transfer characteristics of the same device in the linear scale before and after applying the cap. The threshold voltage $\left(V_{t h}\right)$ is calculated from the $\mathrm{x}$-intercept of the linear fit in the strong inversion region (dotted lines in Figure $4 \mathrm{~b}$ ). The positive shift in $V_{t h}$, observed in all measured devices after applying the cap, is attributed to the net impact of the local dipole electric field and the back gate electric field. Since $\mathrm{MoS}_{2}$ works like an n-type FET, this leads to a lower density of carriers in the device channel. The inset in Figure $4 \mathrm{~b}$ shows the drain current $\left(I_{d}\right)$ for the same overdrive gate bias. The reduction of $I_{d}$ at the same overdrive gate bias after applying the cap, is likely due to the local electric field caused by the alignment of the dipoles of the cap. As a result of this local electric field the density of the charge carriers and the drain current decreases. 

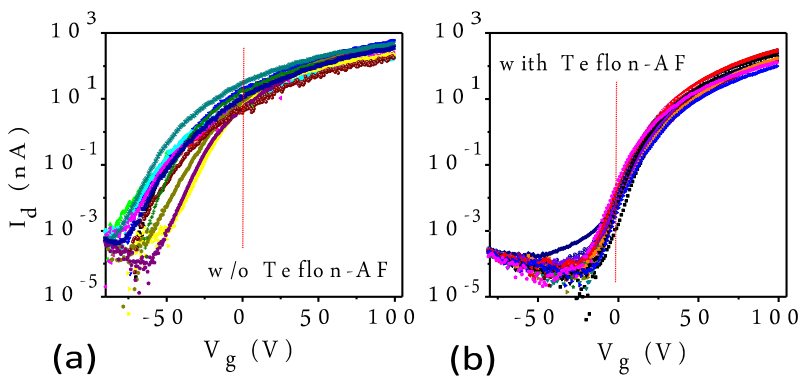

Figure 3. Transfer characteristics of representative FETs before (a); and after (b) applying Teflon-AF. The $y$-axis is $I_{d}$ in $n A$ unites for both cases and the dotted lines are visual guides emphasizing the $V_{t h}$ shift after applying the polymer cap.
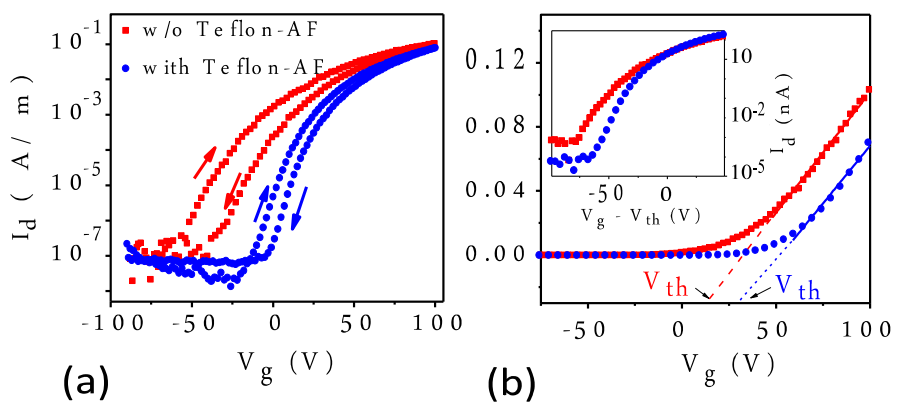

Figure 4. (a) Transfer curves shows a reduction in the gate hysteresis and the desirable enhancement mode character of the transistor after applying the polymer cap; (b) Transfer curves plotted in the linear scale shows a positive $V_{t h}$ shift after applying the polymer cap that could be explained either by the trapped charges between the cap and channel or hole doping of the channels. The inset shows reduction of off-state current and slight improvement of on-state current at high overdrive gate bias after applying the cap.

In order to study the impact of Teflon-AF, approximately 60 devices were tested under ambient conditions before and after applying the cap. The distribution of threshold voltage $\left(V_{t h}\right)$, gate hysteresis, subthreshold swing and current on-off ratio of the tested devices are shown in Figures $5 a, b$ and $6 a, b$. The average value of the $V_{\text {th }}$ shifts from $15.8 \mathrm{~V}$ to $58 \mathrm{~V}$ after applying the cap. This leads to about two orders of magnitude reduction of the drain current at $V_{g}=0 \mathrm{~V}$ and effectively turning off the $\mathrm{MoS}_{2}$ FET at zero back gate bias.
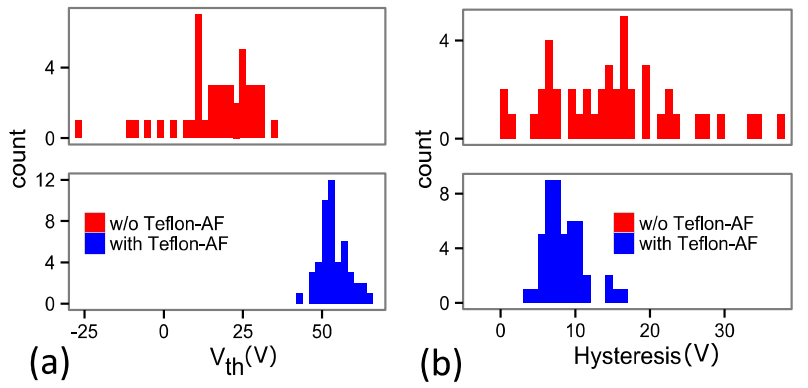

Figure 5. Histogram distribution of (a) threshold voltage; (b) gate hysteresis of 60 measured devices before and after applying Teflon-AF cap. 


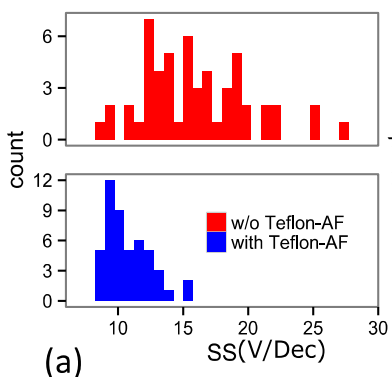

(a)

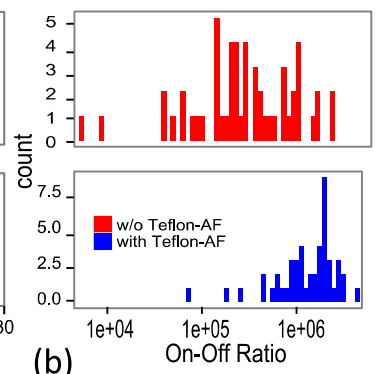

(b)

Figure 6. Histogram distribution of (a) subthreshold swing; and (b) On-Off ratio of 60 measured devices before and after applying Teflon-AF cap presented in a log-scale $x$-axis.

Figure $5 \mathrm{~b}$ presents the distribution of gate hysteresis. The hysteresis is calculated by taking the difference between the $V_{\text {th }}$ when $V_{g}$ is swept from negative to positive direction and vice versa. An improvement was observed in both the average and the standard deviation of the gate hysteresis distribution after applying the cap. The average value reduces from $15.1 \mathrm{~V}$ to $8 \mathrm{~V}$. The gate hysteresis is mainly caused by the trapped mobile charges present within the gate dielectric and at the interface of the channel that rearrange under gate bias and drain voltage. The polar structure of Teflon-AF proved significantly effective in neutralizing the mobile charge traps.

Figure 6a presents the subthreshold swing (SS) of tested devices calculated using, $S S=\ln 10 \frac{d V_{g}}{\operatorname{dln}\left(I_{d}\right)}$. The average value of $S S$ reduces from $15.9 \mathrm{~V} / \mathrm{Dec}$ and $10.7 \mathrm{~V} / \mathrm{Dec}$ after applying the cap. This reduction is in agreement with reduced charge transfer between the surface adsorbates and channel carriers. The reduction of the standard deviation of all three performance metrics here shows an improved device to device consistency.

Figure $6 \mathrm{~b}$ presents the improved on-off switching ratio after applying Teflon-AF. The average value changes from $5 \times 10^{5}$ to $2 \times 10^{6}$ after applying the cap, showing a significant improvement of off-current state.

\section{Conclusions}

In conclusion, we report the improved performance of $\mathrm{MoS}_{2}$ FETs by applying a fluoropolymer capping layer. Our results show a significant improvement in device performance variability, gate hysteresis, subthreshold swing and on-off ratio. These improvements are attributed to desorption of ambient adsorbates from the surface of the channel and the gate dielectric and also the polar nature of the fluoropolymer that neutralizes the impact of charge impurities.

Acknowledgments: This work was supported in part by the NSF CAREER award (D.A.), the NSF-NASCENT Engineering Research Center (Cooperative Agreement No. EEC-1160494), and the Nanoelectronics Research Initiative (NRI) Southwest Academy of Nanoelectronics (SWAN) Center. The fabrication was conducted at the Microelectronics Research Center (MRC) at the University of Texas at Austin, which is a member of the National Nanotechnology Coordinated Infrastructure Network (NNCI).

Author Contributions: S.R. and R.G. contributed equally to this work. S.R. and R.G. conceived and designed the experiments; R.G. was responsible for material synthesis and characterization, S.R. was responsible for device fabrication, characterization and analysis. S.K. was responsible for fluoropolymer application to the transistors. S.R., R.G., S.K., A.D., S.B. and D.A. wrote the manuscript together.

Conflicts of Interest: The authors declare no conflict of interest.

\section{References}

1. Frey, G.L.; Elani, S.; Homyonfer, M.; Feldman, Y.; Tenne, R. Optical-absorption spectra of inorganic fullerenelike $\mathrm{MS}_{2}$. Phys. Rev. B 1998, 57. [CrossRef]

2. Mak, K.F.; Lee, C.; Hone, J.; Shan, J.; Heinz, T.F. Atomically thin $\mathrm{MoS}_{2}$ : A new direct-gap semiconductor. Phys. Rev. Lett. 2010, 105. [CrossRef] [PubMed] 
3. Cao, T.; Wang, G.; Han, W.P.; Ye, H.Q.; Zhu, C.R.; Shi, J.R.; Niu, Q.; Tan, P.H.; Wang, E.; Liu, B.L.; et al. Valley-selective circular dichroism of monolayer molybdenum disulphide. Nat. Commun. 2012, 3. [CrossRef] [PubMed]

4. $\quad$ Bhimanapati, G.R.; Lin, Z.; Meunier, V.; Jung, Y.; Cha, J.J.; Das, S.; Xiao, D.; Son, Y.; Strano, M.S.; Cooper, V.R.; et al. Recent advances in two-dimensional materials beyond graphene. ACS Nano 2015, 9, 11509-11539. [CrossRef] [PubMed]

5. Radisavljevic, B.; Whitwick, M.B.; Kis, A. Integrated circuits and logic operations based on single-layer $\mathrm{MoS}_{2}$. ACS Nano 2011, 5, 9934-9938. [CrossRef] [PubMed]

6. Radisavljevic, B.; Whitwick, M.B.; Kis, A. Small-signal amplifier based on single-layer MoS 2 . Appl. Phys. Lett. 2012, 101. [CrossRef]

7. Zeng, H.; Dai, J.; Yao, W.; Xiao, D.; Cui, X. Valley polarization in $\mathrm{MoS}_{2}$ monolayers by optical pumping. Nat. Nanotechnol. 2012, 7, 490-493. [CrossRef] [PubMed]

8. Ghorbani-Asl, M.; Zibouche, N.; Vahiduzzaman, M.; Oliveira, A.F.; Kuc, A.; Heine, T. Electromechanics in $\mathrm{MoS}_{2}$ and $\mathrm{WS}_{2}$ : Nanotubes vs. monolayers. Sci. Rep. 2013, 3. [CrossRef] [PubMed]

9. Nayak, A.P.; Pandey, T.; Voiry, D.; Liu, J.; Moran, S.T.; Sharma, A.; Tan, C.; Chen, C.H.; Lee, L.J.; Chhowalla, M.; et al. Pressure-dependent optical and vibrational properties of monolayer molybdenum disulfide. Nano Lett. 2015, 15, 346-353. [CrossRef] [PubMed]

10. Sanne, A.; Ghosh, R.; Rai, A.; Yogeesh, M.N.; Shin, S.H.; Sharma, A.; Jarvis, K.; Mathew, L.; Rao, R.; Akinwande, D.; Banerjee, S. Radio frequency transistors and circuits based on CVD MoS 2 . Nano Lett. 2015, 15, 5039-5045. [CrossRef] [PubMed]

11. Chang, H.-Y.; Yogeesh, M.N.; Ghosh, R.; Rai, A.; Sanne, A.; Yang, S.; Lu, N.; Banerjee, S.; Akinwande, D. Large-area monolayer $\mathrm{MoS}_{2}$ for flexible low-power RF nanoelectronics in the GHz regime. Adv. Mater. 2016, 28, 1818-1823. [CrossRef] [PubMed]

12. Rahimi, S.; Tao, L.S.N.; Chowdhury, Sk.F.; Park, S.; Jouvray, A.; Buttress, S.; Rupesinghe, N.; Teo, K.; Akinwande, D. Toward $300 \mathrm{~mm}$ wafer-scalable high-performance polycrystalline chemical vapor deposited graphene transistors. ACS Nano 2014, 8, 10471-10479. [CrossRef] [PubMed]

13. Kang, K.; Xie, S.; Huang, L.; Han, Y.; Huang, P.Y.; Mak, K.F.; Kim, C.J.; Muller, D.; Park, J. High-mobility three-atom-thick semiconducting films with wafer-scale homogeneity. Nature 2015, 520, 656-660. [CrossRef] [PubMed]

14. Wang, H.; Wu, Y.; Cong, C.; Shang, J.; Yu, T. Hysteresis of electronic transport in graphene transistors. ACS Nano 2010, 4, 7221-7228. [CrossRef] [PubMed]

15. Franklin, A.D.; Tulevsky, G.S.; Han, S.J.; Shahrjerdi, D.; Cao, Q.; Chen, H.Y.; Wong, H.-S.P.; Haensch, W. Variability in carbon nanotube transistors: Improving device-to-device consistency. ACS Nano 2012, 6, 1109-1115. [CrossRef] [PubMed]

16. Davis, S.M.; Carver, J.C. Oxygen chemisorption at defect sites in $\mathrm{MoS}_{2}$ and $\mathrm{ReS}_{2}$ basal plane surfaces. Appl. Surf. Sci. 1984, 20, 193-198. [CrossRef]

17. Jaegermann, W.; Schmeisser, D. Reactivity of layer type transition metal chalcogenides towards oxidation. Surf. Sci. 1986, 165, 143-160. [CrossRef]

18. Qiu, H.; Pan, L.; Yao, Z.; Li, J.; Shi, Y.; Wang, Z. Electrical characterization of back-gated bi-layer $\mathrm{MoS}_{2}$ field-effect transistors and the effect of ambient on their performances. Appl. Surf. Sci. 2012, 100. [CrossRef]

19. Chowdhury, Sk.F.; Sonde, S.; Rahimi, S.; Tao, L.; Banerjee, S.; Akinwande, D. Improvement of graphene field-effect transistors by hexamethyldisilazane surface treatment. Appl. Surf. Sci. 2014, 105. [CrossRef]

20. Lafkioti, M.; Krauss, B.; Lohmann, T.; Zschieschang, U.; Klauk, H.; Klitzing, K.V.; Smet, J.H. Graphene on a hydrophobic substrate: Doping reduction and hysteresis suppression under ambient conditions. Nano Lett. 2010, 10, 1149-1153. [CrossRef] [PubMed]

21. Ha, T.J.; Lee, J.; Chowdhury, Sk.F.; Akinwande, D.; Rossky, P.J.; Dodabalapur, A. Transformation of the electrical characteristics of graphene field-effect transistors with fluoropolymer. ACS Appl. Mater. Interfaces 2012, 5, 16-20. [CrossRef] [PubMed]

22. Jang, S.; Kim, B.; Geier, M.L.; Prabhumirashi, P.L.; Hersam, M.C.; Dodabalapur, A. Fluoropolymer coatings for improved carbon nanotube transistor device and circuit performance. Appl. Surf. Sci. 2014, 105. [CrossRef]

23. Kim, J.; Liu, Y.; Zhu, W.; Kim, S.; Wu, D.; Tao, L.; Dodabalapur, A.; Lai, K.; Akinwande, D. Toward air-stable multilayer phosphorene thin-films and transistors. Sci. Rep. 2015, 5. [CrossRef] [PubMed] 
24. Lee, Y.; Zhang, X.; Zhang, W.; Chang, M.; Lin, C.; Chang, K.; Yu, Y.; Wang, J.; Chang, C.; Li, L.; et al. Synthesis of large-area $\mathrm{MoS}_{2}$ atomic layers with chemical vapor deposition. Adv. Mater. 2012, 24, 2320-2325. [CrossRef] [PubMed]

25. Splendiani, A.; Sun, L.; Zhang, Y.; Li, T.; Kim, J.; Chim, C.Y.; Galli, G.; Wang, F. Emerging photoluminescence in monolayer $\mathrm{MoS}_{2}$. Nano Lett. 2010, 10, 1271-1275. [CrossRef] [PubMed]

26. Tavana, H.; Hair, M.L.; Neumann, A.W. Influence of electronic properties of naphthalene compounds on contact angles. J. Phys. Chem. B 2006, 110, 1294-1300. [CrossRef] [PubMed]

27. Yang, M.K.; French, R.H.; Tokarsky, E.W. Optical properties of Teflon ${ }^{\circledR}$ AF amorphous fluoropolymers. J. Micro/Nanolithogr. MEMS MOEMS 2008, 7. [CrossRef]

28. Park, W.; Park, J.; Jang, J.; Lee, H.; Jeong, H.; Cho, K.; Hong, S.; Lee, T. Oxygen environmental and passivation effects on molybdenum disulfide field effect transistors. Nanotechnology 2013, 24. [CrossRef] [PubMed]

29. Namgung, S.D.; Yang, S.; Park, K.; Cho, A.-J.; Kim, H.; Kwon, J.-Y. Influence of post-annealing on the off current of $\mathrm{MoS}_{2}$ field-effect transistors. Nanoscale Res. Lett. 2015, 10. [CrossRef] [PubMed]

(C) 2016 by the authors; licensee MDPI, Basel, Switzerland. This article is an open access article distributed under the terms and conditions of the Creative Commons Attribution (CC-BY) license (http://creativecommons.org/licenses/by/4.0/). 